\title{
AMBUBOT: Ambulance Robot Automated External Defibrillator Robotic Ambulance
}

\author{
Mohammad Arif*, Hooman Samani* \\ *Department of Electrical Engineering, National Taipei University, Taiwan \\ arifayip91@gmail.com, hooman@mail.ntpu.edu.tw
}

\begin{abstract}
The purpose of this paper is to introduce a novel robot in order to overcome some difficulties in providing an Automated External Defibrillator (AED) device at the nearest location of victim suffering from sudden cardiac arrest in the shortest possible time before the advent of the ambulance. We designed and developed an ambulance robot, called it Ambubot, that brings along an AED to help lay rescuers for saving patients life in a sudden event of cardiac arrest. The first aid to the victim can be carried out once an incident alarm is transmitted to the Ambubot station by sensing via body-attached sensor and/or mobile phone application. Such applications transmit required information to the Ambubot center for further execution. Ambubot center is integrated with three independent servers namely database server, message controller, and GIS server. In addition, message controller server is connected with telecom's short message server for transmitting the message to family members of the patient. The Ambubot robots are located in Ambubot stations when several stations can be covered via single center where human operators are located. Different methods had been proposed for dispatching Ambubot to locate the victim namely tele-control, partially autonomous, and fully autonomous operations. While we have illustrated all those methods in this paper we present the conduction of the tele- control method to control the operation of the robot. In this method, not only Ambubot follows instructions of human operator till the robot reaches the location of victim and delivers the AED but also provides instruction to the people in the location for applying the AED hence the lay rescuers will dry the victim's chest and attach the AED pads by themselves while instructed and monitored by the human experts in the main center in real-time.
\end{abstract}

Keywords — Network Robotic, Tele-Robot, Medical Robot, Telematics, Rescue Robot.

\section{INTRODUCTION}

Emergency situation refers to any unforeseen event that can jeopardize and bring significant injuries on a person's life. This situation can be broken down into two basic categories, natural and manmade calamities. Natural calamity is the phenomena of nature caused by environmental factors that can bring catastrophic consequences. While the world population grows rapidly with increasing their concentration in hazardous environments without giving much consideration to the local geo-climatic conditions have exacerbated the devastation caused by natural calamities. Therefore, different forms of natural calamities like drought, earthquake, extreme temperature, mass movement wet, typhoon, and volcano strike according to the vulnerability of the area in the globe. On the other hand, the catastrophe can also be caused as the consequence of technological or human hazards, including industrial accident and transport accident, where it is commonly known as manmade calamity.

Natural calamity is generally inevitable and ubiquitous worldwide that kills thousands of people and destroys billions of dollars of habitat and property each year. According to The International Disaster Database (EM-DAT)'s data [1], the number of deaths reported is getting less, however the number of people reported affected by natural calamity is increasing dramatically. It was due to global warming and the fact that the technology advancement makes the frequency of global calamity tends to be higher. Therefore, decreasing the rate of mortality is usually seen as the most effective way.

TABLE 1. RESPONSE TIME OF AMBULANCE SERVICES IN VARIOUS TERRITORIES

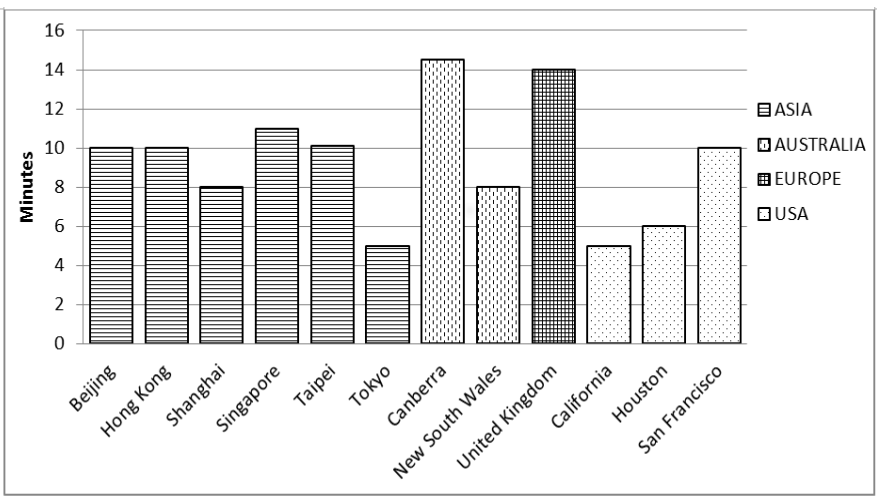

We typically dial a three-digit number for ambulance services when there are sick or injured person needing an immediate response. Once a call is identified as emergent in nature, the ambulance will be dispatched to the scene in average of ten minutes time [2]. Details of that information are depicted in Table 1 for various territories. In practice, the advent time of ambulance is far above the ten minutes standard in many cases. This is mainly due to many 
obstructions during the process of dispatching and it may defer the patient from receiving the service on time. The delay is largely subjected on a various factors such as the long distance, traffic congestion, and difficulty to locate the address. Any one of these delays can lead to increase in response time.

The ambulance has to transport the patient to the hospital as quickly and safely as possible. However, in many cases like life-threating emergencies the patient needs immediate first aid and medical attention to prevent serious danger. Meanwhile, the fate of patients cannot be influenced by waiting the ambulance but rather could be changed if some treatments could be given within a few minutes of the patient's collapse. For instance, individuals suffering sudden cardiac arrest could be saved if the Automated External Defibrillator device known as AED is applied within a few minutes after the occurrence of cardiac arrest. At the same time, someone who helps the patient must be able to perform CPR (Cardiopulmonary Resuscitation) and attaches an AED to a person in cardiac arrest. The AED is small electronic portable defibrillator that is designed for minimally trained or untrained non-medical personnel [3]. This device can generate single-phase and double-phase waveforms. Single-phase waveforms generate a high-energy output. It may cause damage to the heart and skin. In contrary, double-phase waveforms produce a low energy output. This output shocks heart twice at the same time and decreases the complication caused by the defibrillator.

According to the guideline of using the AED, the helping person needs to call for ambulance immediately even before applying the AED. In the case of existence of two helping people, one is advised to call for ambulance while the other one is dealing with the AED. Though AEDs are deemed as medical devices, yet lay people can use these. However, it would be better if it were doing by someone who has completed a first aid training course. Despite the fact that AEDs are located in many public places, in practice it is difficult for people to find these in an emergency situation. It is due to the initial panic that often occurs when people are faced in such circumstances. In order to mitigate those problems and keep patient staying alive before the advent of ambulance, we propose an idea of using Ambubot (Ambulance Robot) that could bring an AED and according to our long-term plan it would be able to perform CPR to a person in cardiac arrest, which is shown in Figure 1.

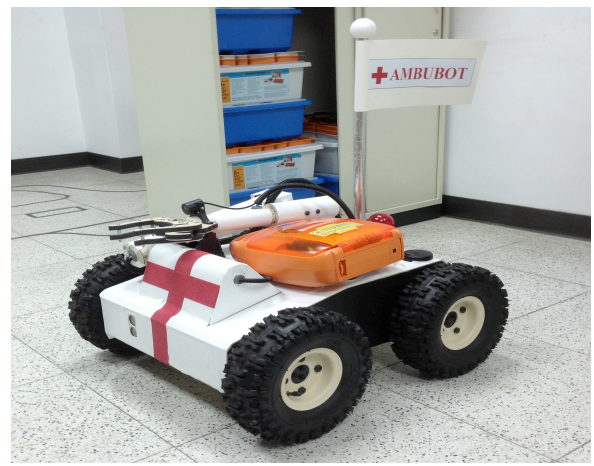

Figure 1. The Ambulance Robot (Ambubot)
We believe that our application can mitigate the constraint of human ability to locate the AED at the nearest location of victim and increase the survival time of victim. In the following of the paper, we will describe more details about this research. Rescue and medical robots are explicitly described in section 2. The detailed description of the system is presented in Section 3. In Section 4, tele-control method is discussed in details. Finally, we conclude with the future works in this research.

\section{BACKGROUND}

Due the natural and manmade calamities, developments of medical and rescue robots are essential. This section will provide a brief description of rescue and medical robots in terms of both the size and function of robots in distinct environments. In addition, it will include how and which types of robots can be applied in various situations.

\section{A. Rescue Robots}

Most rescuers occur shortly after the event of a calamity happens. In that event, human rescuers will organize the rescue planning to get out to the calamity areas, find victims, and help them as fast as possible. They have very short time to find victims in any calamity situation; otherwise the likelihood of finding victims still alive is nearly zero. In such a critical situation, technology can be used to support rescuers in different tasks. Intelligent mobile robots and cooperative multi-agent robotic systems are increasingly being used in many different ways to find and save victims in a faster and more efficient way. The robot that can do such tasks is well known as rescue robot. Rescue robot is a robot that has been precisely designed to do rescuing jobs in situations that are hazardous for mankind to handle it, for instance rainstorms, collapsed buildings, obstructions, and dangerous substances.

To be useful tools rescue robots have to be fairly small, not too heavy and maneuverable enough to enter into gaps and move through cracks or narrow spaces that are impossible for humans and even trained dogs. These robots must be capable of navigating in challenging situation both indoors and outdoor terrain to find victims. Typically, robots will be equipped with tele-operated so that they have a good communication with human rescuers to gather crucial information including the location of victims in a map and way that human rescuers can reach victims. It can also place a small package containing food, medication, and a communication device near the victim.

Rescue robots are broadly divided into four different categories depending on modality and model size. There are four different groups of the robot based on modalities namely UGVs (Unmanned Ground Vehicles), UAVs (Unmanned Aerial Vehicles), UUVs (Unmanned Underwater Vehicles), and USVs (Unmanned Surface Vehicles) [4]. UGVs are typically placed on the ground to help human rescuers to find victims in areas that too dangerous for human. UAVs are developed to transport medical treatments to victims. These robots can extract meaningful information about the surrounding conditions to the responders. Water-based robot 
also called as UUVs are designed to replace humans for working underwater where it is both dangerous and difficult for humans. USVs have ability to work on the water surface. These robots can help rescuers to locate and bring some equipment to victims.

Rescue robots can further be divided in three groups, depending on the model sizes, man-packable, man-portable, and maxi-sized. Man-packable robots are typically small and more likely to be used immediately after a calamity. These robots can also travel over debris and climb stairs into a calamity hot zone. The next larger size is man-portable robots that may need two people or a small terrain vehicle. Manportable robots are often used for logistics support both in the hot zone and outside the hot zone. Maxi-sized robots are robots with the biggest size and need trailer or other special transportation logistics.

Rescue robots had been used in the last few decades with the participation in the recent devastations, such as 2001 the World Trade Center (WTC) collapse, the 2004 Mid Niigata earthquake in Japan, the 2005 hurricanes Katrina in the United States, as well as the 2011 Tohoku earthquake and tsunami in Japan. Three species of small UGVs namely Inuktun microVGTV, Inuktun micro-Tracks, and Foter-Miller Solem were sent to find victims at the World Trade Center 9/11 disaster in New York City, United States [5]. In the former incident robots were used to explore and inspect the rubble of the twin towers.

On 23 October 2004 the largest earthquake hit the Niigata Chuetsu in Japan and thirty-nine people were reported dead. The first snake robot of the Soryu III has been developed to find victims at a calamity site and it had a length of $1.2 \mathrm{~m}$, a weight about $10 \mathrm{~kg}$, and a maximum speed of $0.37 \mathrm{~m} / \mathrm{s}$ [6]. There are three components mounted upon the robots namely a charge-coupled device (CCD) camera, an infrared camera (FLIR), and two-way audio as well as proprioceptive sensors. This robot is also supported with $\mathrm{CO}_{2}$ sensor to detect human breathing. The worst hurricane has been happened in the southern Gulf Coast of the United States in 2005 and the 2000 lives reported to have died [7]. There were two UAVs used to get information about rural regions cut off by flash flooding in the aftermath of calamities.

Since some incidents of the aforementioned have attacked in some part of the globe, rescue robots have been widely used to help victims at calamities site where human rescuers cannot enter. Such a situation was realized at the Fukushima nuclear reactors in Japan after the Higashi Nippon earthquake that occurred on 11 March 2011. The earthquake destroyed the interiors of buildings and causing some areas were highly contaminated by radiation. Several types of robots have been used to explore areas inside the reactor buildings where humans could not enter such as Packbot [8], and Quince [9].

\section{B. Medical Robots}

Nowadays, most of robots produced are typically created for industrial sectors. These robots have replaced human laborers involvement in hazardous and harmful tasks. The number of robots used in industrial production has grown strongly within the past few decades. On the other side, the development of robots in service sectors is still limited. These robots are often called as service robots that perform tasks for people instead of serving industrial manufacturer. Service robots are often mobile, capable of working independently, and interacting with humans. These robots are comprised of various types and one of them is a medical robot. In the recent years, application of robots in medicine has become a more interesting topic for both robotics researchers and the general public. They have been used to fundamentally change interventional medicine with robots and bring some new techniques to support physical therapy, rehabilitation, and even perform more difficult procedures.

Medical robots can be classified into two categories follow their utilization which are medical robots to assist people and medical robots to assist medical staffs. There are two distinct groups of robots that typically used for assisting people, such as rehabilitation and companion robots. Rehabilitation robots are dedicated to provide assistive equipment for those people with impairment following stroke. Hybrid Assistive Limb 5 [10], Lokomat [11], and Bionic Limbs [12] are most widely used in hospitals for rehabilitation. Companion robots offer tremendous potential for enhancing the quality of human lives in the future. There are a several examples of companion robotics, such as Lovotics [13], RIBA [14], and Paro Robot [15].

In addition, there are three different groups of robots that typically used for assisting medical staffs, such as telerobotics, surgical, and pharmacy robots. Tele-robotics is enabling many participants to control the robot simultaneously that are situated in remote location or otherwise inaccessible. The list of tele-robotics that helps in medicine is including Remote Presence-Vita Robot [16] and Nursebot. Surgical robotics can be categorized into two classes, which are robots that perform surgery, e.g. Cyberknife Radiosurgery [17], and robots that assist the surgeon in the surgery, e.g. Da Vinci robot [18]. Pharmacy robots can handle a wide range of tasks for preparing intravenous medications in a sterile environment such as packaging, and labeling of medications.

\section{SYSTEM DESCRIPTION}

Sudden cardiac arrest is the leading cause of death worldwide. It can happen anywhere at work, at home or anywhere else. AEDs are designed to help someone in cardiac arrest. However, it may take a long time to get an AED at nearest scene of victims because AEDs are not available everywhere. Therefore, we have proposed the Ambubot as a platform to save someone's life during cardiac arrest. This robot can be characterized by different degree of autonomy and it will execute different tasks as illustrated in Table 2.

TABLE 2. DIFFERENT TASKS OF AMBUBOT

\begin{tabular}{|l|c|c|c|}
\hline \multicolumn{1}{|c|}{ Task } & $\begin{array}{c}\text { Tele- } \\
\text { control }\end{array}$ & $\begin{array}{c}\text { Partially } \\
\text { Autonomous }\end{array}$ & Autonomous \\
\hline $\begin{array}{l}\text { Autonomous } \\
\text { Navigation }\end{array}$ & No & Yes & Yes \\
\hline $\begin{array}{l}\text { Execute the } \\
\text { AED }\end{array}$ & No & No & Yes \\
\hline
\end{tabular}




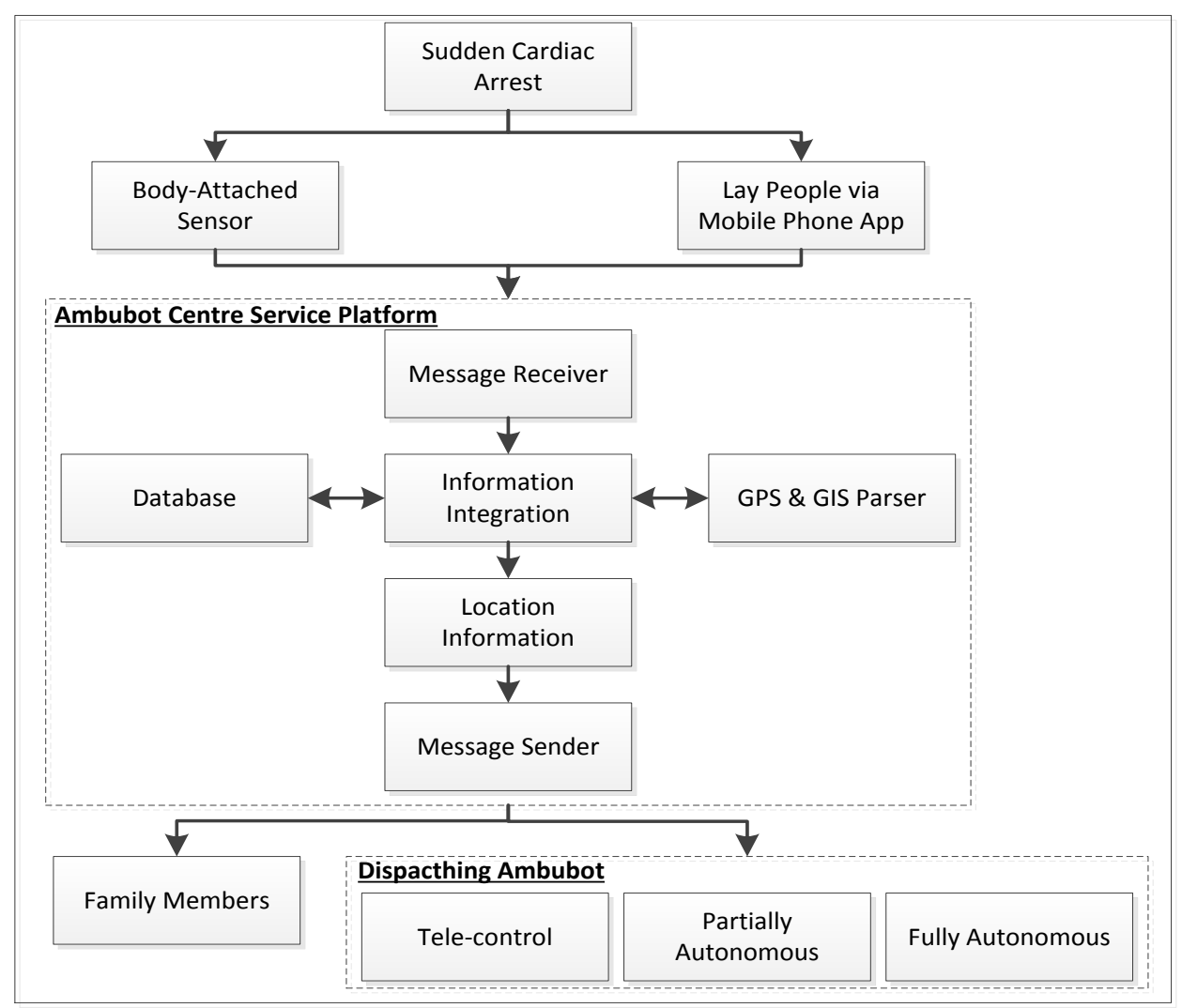

Figure 2. Overview of the system workflow

As shown in Figure 2, there are two techniques that can be used to keep cardiac arrest victims alive either by bodyattached sensor or mobile phone application. Whenever one of them is used, they will immediately send out warning message and Global Positioning System (GPS) information to the Ambubot center. The Ambubot center will convert the longitude and latitude coordinates into a street map location using a GPS and GIS parser. Due the body-attached sensor acceptable as fall sensor, this location will be integrated with other basic information such as personal contacts and characteristics, blood type, height, weight, and photograph to generate the complete information needed for helping victims. After the Ambubot center processes this data packet, it will generate two commands namely a command for dispatching Ambubot from the station to the scene as precaution to save patient life before ambulance arrives and other command for delivering an emergency message to family members via Global System for Mobile Communication (GSM) so that they can obtain relevant information concerning the falling person via mobile phone. Family members will be alerted through this message in case of victims have mounted with the bodyattached sensor. We also consider informing ambulance from the nearest hospital after confirmation of the incident. The Ambubot can move with high speed and accuracy to the location of victim. It can mitigate the lateness problems that always facing by ambulance.
The hardware of the body-attached sensor mainly consists of a GPS satellite location module, a gyro sensor, a microprocessor, and a GSM communication module as depicted in Figure 3. The body-attached sensor will be integrated in the objects that a person frequently uses (e.g., glasses frames, belts, and watches) to give a convenience without disturbing the person' daily lives. The dimension of the body-attached sensor is suitable for the fixation on the human body and produces low power consumption.

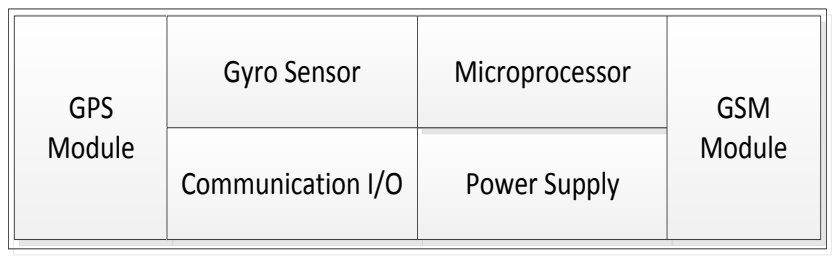

Figure 3. Block diagram of body-attached sensor

The GPS module is used to provide satellite location information needed to find the shortest path of victims, such as longitude/latitude coordinates and direction. The gyro sensor is integrated with accelerometer $\mathrm{x}, \mathrm{y}$, and $\mathrm{z}$ thus acceptable as fall sensor. This sensor will be concatenated with tag that contains the patient identification code. A patient can be identified by identification code once registered. Information including the patient's name, date of birth, age, photographs, relatives contact information and personal health 
history will be generated by the Ambubot center to help ensure the patient's safety. The microprocessor is in charge of computation as the main intelligent hardware module. The GSM communication module is used to provide a communication channel to transmit emergency rescue messages concerning the patient to the Ambubot center and receive commands from the server. Since the body-attached sensor requires a certain amount of power to function properly, it is important to have a power supply that can provide the right amount. When $10 \%$ of the power is left, this function sends a message to family members to replace the battery so as to ensure the normal operation of the system. There are various ways of dispatching Ambubot to reach a destination, such as tele-control, partially autonomous and fully autonomous. The details of these methods are described in details as following.

\section{A. Tele-control}

Tele-control assists a human operator to direct maneuvering the Ambubot using a visual display and a control pad. The overview of the proposed tele-control system is depicted in Figure 4. In general, the main function of the tele-control system is to assist the human operator to perform and accomplish complex, uncertain tasks in hazardous, and less structured environments. In this method, an Ambubot needs a people like driver who will control the robot by using a remote control device that resembles a controller panel and watch the real-time video stream from two surveillance cameras on the Ambubot to navigate, locate, and approach the victim. In this scenario, when the Ambubot approaches the victims, the human operators from the control center provide detail instruction to the people in the vicinity of the victim to operate the AED device that was delivered by Ambubot.

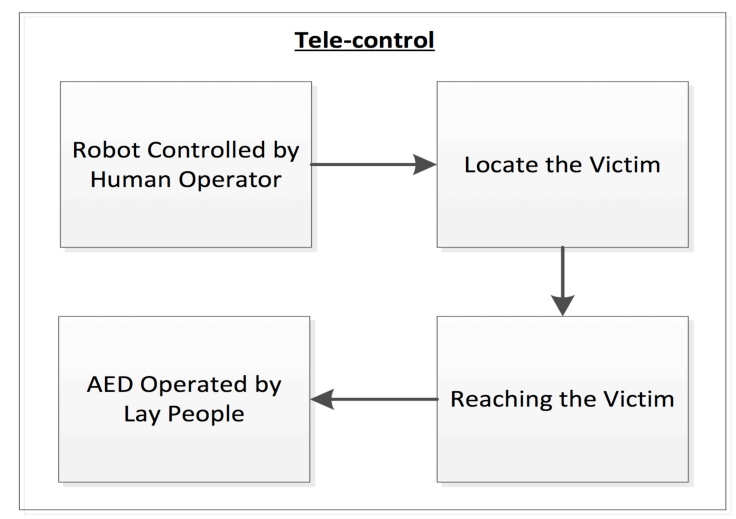

Figure 4. Workflow of the search assistance for tele-control

\section{B. Partially Autonomous}

In this part, the task execution of Ambubot utilizes both autonomous functions of the robot and direct maneuvering by the human operator. The flexibility and human intelligence become key factors in controlling the robot for partially autonomous. It is due to the robot is not intelligent enough to make complete task plans. Safety issue is another concern. Hence, the human operator should help in accommodating task procedures to the real environment. As depicted in Figure
5, humans near the victims still play an important role to apply the pads of AED to the victim's chest. When the Ambubot center receives the GPS information regarding the victim, the main server located in the server computes the shortest path and transmit it to the robot. Ambubot will carry an AED and follow the path for tracing the victim until the destination has been found. In this stage Ambubot is in autonomous navigation and obstacle avoidance mode. Ambubot reports the current situation and displays the motion through streaming the video using two cameras mounted on the robot, one in front of the body and the other one on the arm. Such operation method would improve the task of navigation compare to the manual mode. Additionally, it may help to reduce the stress on the operators because they can easily understand what the robot is going to do and how to send data to the robot. Although there is no human intervention for controlling the robot but the operator assistance still needed to deliver some additional information to the robot based on the circumstances.

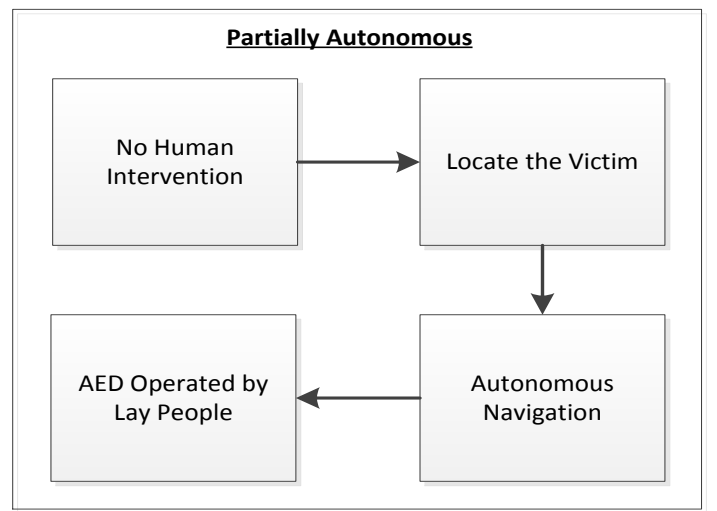

Figure 5. Workflow of the search assistance for partially autonomous

\section{Fully Autonomous}

While it was shown that dispatching of the Ambubot can be performed in tele-control or partially autonomous, as the most advanced method, it can have other practical use for delivering an AED to the location of victim through a fully autonomous navigation and the AED operation.

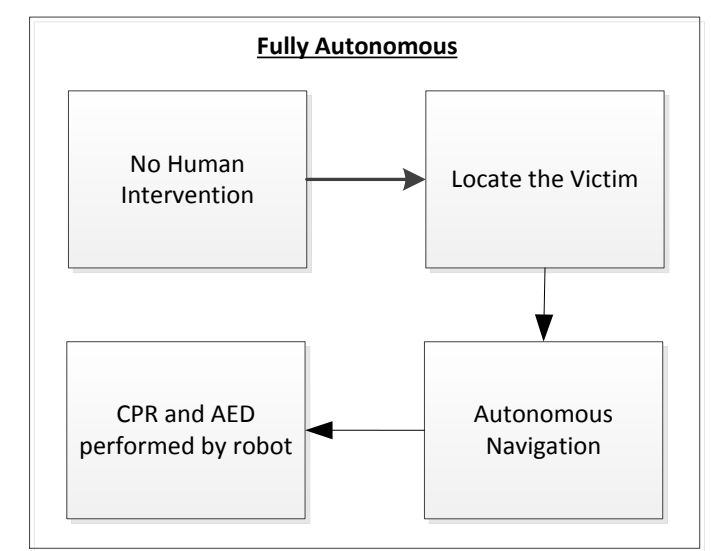

Figure 6. Workflow of the search assistance for fully autonomous 


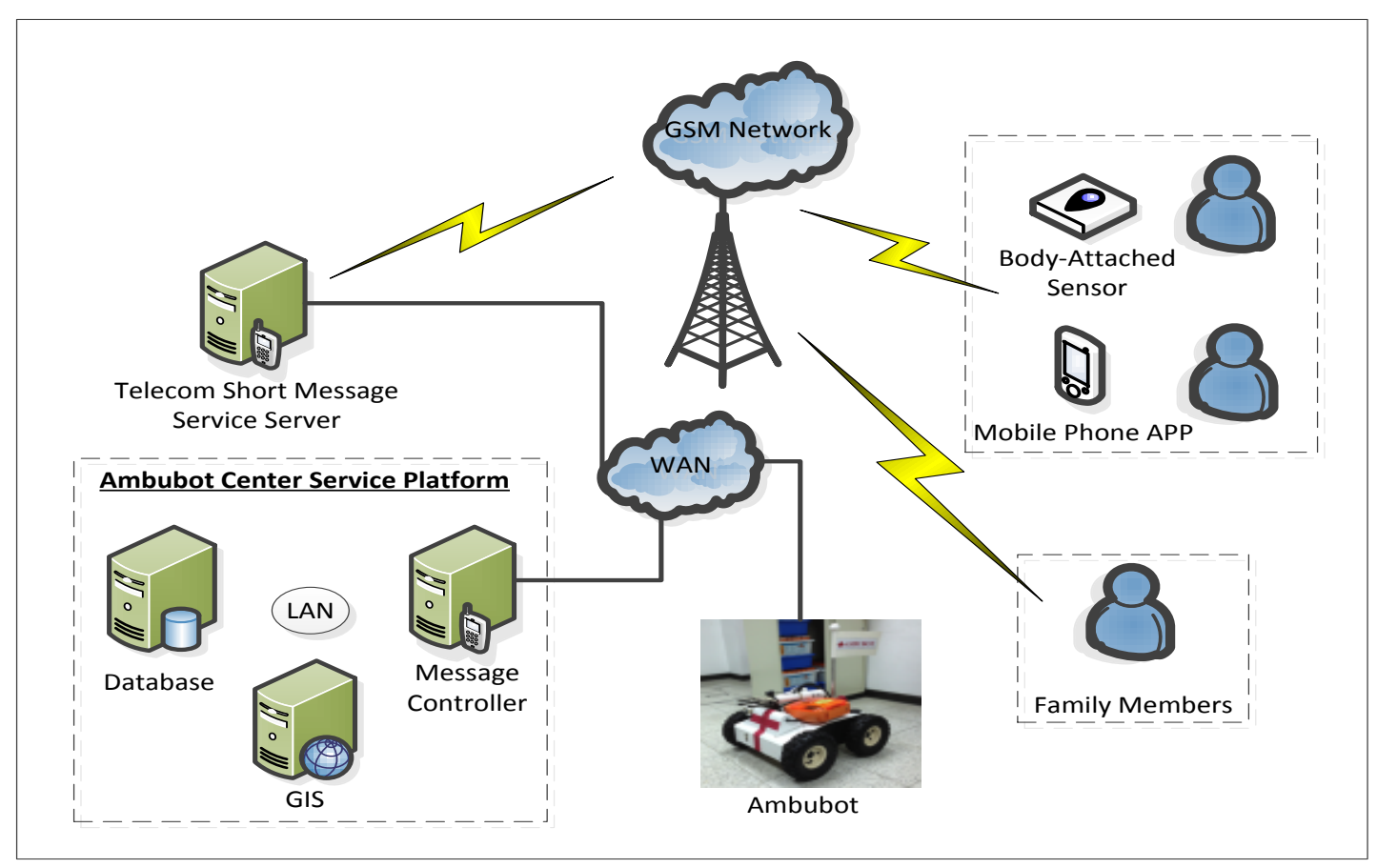

Figure 7. System Architecture of Ambubot center

The main difference lies on the execution of AED. In the previous sections, lay rescuers apply the pads of AED by themselves on the victim's chest. However, in this method, the Ambubot will execute the AED by itself without continuous human guidance as shown in Figure 6. Also, in our future plan, the Ambubot will perform CPR as the first aid to save victim life in cardiac arrest. Ambubot grabber will execute these two tasks. The grabber has been designed well to be light enough to apply the pads of AED on the victim's chest and strong enough to withstand an AED. One of the challenges here would be in the case that the victim is not in a suitable posture to apply AED.

\section{SYSTEM DESCRIPTION}

In the process of developing Ambubot, we focused on developing the first mode of the Ambubot when it can be used as a tele-existance rescue robot for AED. This system includes the robot located in the station and the control server equipped with computer. Ambubot center service platform consists of three servers that are implemented on three independent server systems namely database server, message controller, and GIS server as shown in Figure 7. All servers are located within a firewall to enhance the system security. Database server is designed for data storage and management. A message controller server is connected to the telecom's short message server for enhancing the efficiency of message processing including the acceptance and transmission of a larger volume of short messages via network packets. The other server is Geographic Information Server (GIS) used to convert the GPS longitude/latitude coordinates to location information in terms of street address and important landmarks, allowing family members and the Ambubot to efficiently acquire geographical spatial information concerning the falling patient and dispatch the Ambubot more effectively. In addition, this server is solely responsible in helping Ambubot to find the shortest path to the location of victim.

Since the system is connected to the GSM network, it will allow the system to overcome the GPS problem in terms of invalid signal. The system will utilize GSM-locating service provided by a local GSM network company to trace the location of victim. Though it was not displaying the location of victim precisely yet it can resolve the problem of not being able to locate the victim in bad weather conditions.

The block diagram in Figure 8 shows the gradual implementation of tele-control system for dispatching Ambubot. Tele-control system provides telepresence by allowing an operator to remotely control the Ambubot to the scene. An emergency message and current position of victim will be generated by both of two applications, which are body attached sensor and mobile phone application. These data will be evaluated and then transmitted automatically to the Ambubot center immediately after a sudden cardiac arrest happens. The majority of people use smart mobile phone. Therefore, the development of mobile phone application for connecting with the Ambubot center is convenient so that it can provide on-time medical care to victims. Alternative method in the case of lack of smart phone would be to call the center. In the Ambubot center, history of victim during cardiac arrest is stored in database server. This is extremely useful for family members to elicit new sight and understand victim's healthcare. When GIS server obtains the location information of victim, this server will track down the location of victim and convert it into important landmarks. It will produce the high precision and accuracy of information about victim's current position. Afterward, history and location information of victim will be integrated with message controller server. 


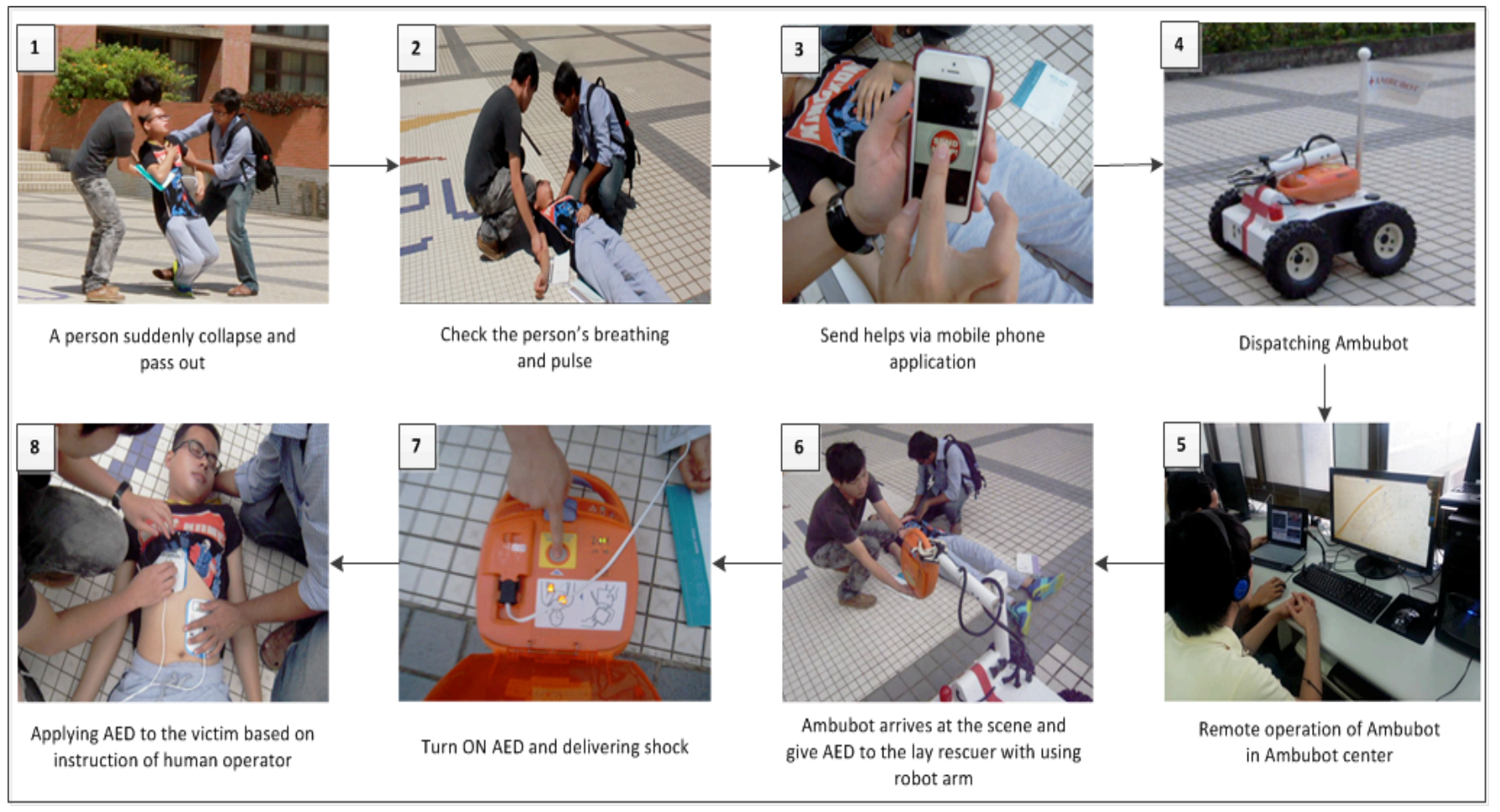

Figure 8. System Architecture of Ambubot center

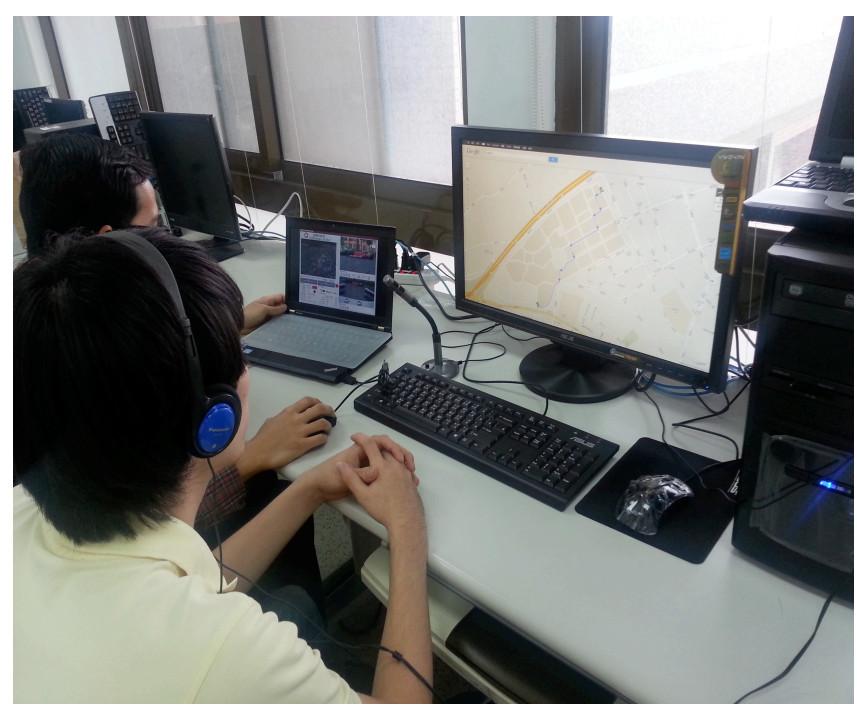

Figure 9. Remote operation of Ambubot in Ambubot center

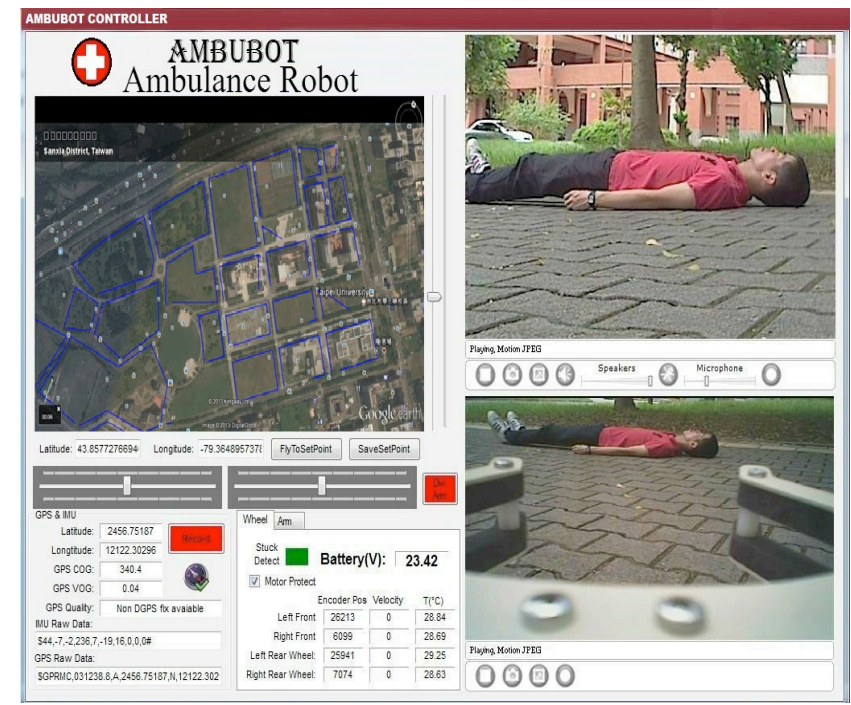

Figure 10. Ambubot controller interface
This server will hook up with telecom's short message server for the process of sending the message to family members. This message combines with other basic information of victim such as personal contacts and characteristics, blood type, height, weight, photograph, and health history of victim in cardiac arrest. After receiving detailed information, an operator will guide the Ambubot to locate the victim, as shown in Figure 9.

The Ambubot has been designed for simple yet fully functional robot that can be used to bring along an AED. With faster maneuverability, this robot can be driven in tough terrains and capable of climbing up the staircases. It integrates outdoor GPS and 9 DOF IMU (Gyro/Accelerometer/Compass) for autonomous navigation. The developed Ambubot is capable of driving up to $10 \mathrm{~km} /$ hour and passing slopes up to 45 degrees [19]. Meanwhile, the high-resolution video and audio is integrated in this robot to provide human operator detail information of the surrounding, as depicted in Figure 10. Human operator will control the robot either by a control pad or a computer. When the Ambubot has arrived in the location of victim, human operator instructs lay rescuers to apply the pads of AED on the victim's chest based on its instruction. 


\section{CONCLUSION \& Future WORKS}

In this paper a novel ambulance robot called Ambubot was presented which provides the service of an ambulance with AED and focus on a tele-operation. Sudden cardiac arrest occurs when the heart has stopped beating effectively due to an electrical malfunction of the heart. It occurs instantly or shortly after symptoms appear. Early access to the AED can be a life saving measure in the event of a person suffering in cardiac arrest. To have the absolute best chance of survival, immediate treatment must be carried out in the first few minutes after a person suffer a sudden cardiac arrest. In this paper, the Ambubot is intended to improve on manual search assistance of finding AED with the help of the information technology so that an immediate treatment can be delivered to help victims in cardiac arrest. There are three dispatching methods of Ambubot to reach location of victim namely telecontrol, partially autonomous, and fully autonomous. However, tele-control robot is a major focus of our current research due to the difficulty of implementing other methods in real health care environment. Such issue is practical where the accident occurs near the Ambubot station. In our case we have focused on the campus of National Taipei University. The Ambubot operator guides a lay first responder through the entire process to apply AED before the ambulance arrives. To help keep the cardiac arrest victims alive, the body-attached sensor and mobile phone application can be used to prevent the victim from suffering any misfortune. For data management, an Ambubot center service platform consisting of database server, message controller, and H-GIS server is constructed to let Ambubot and family members acquire the relevant information about the victim.

Our future efforts will primarily focus on increasing the autonomous operation of the robot while working on two extra aspects. One is adding the ability of the body-attached sensor to monitor condition of the patient and then transmits some helpful information to specialists who would evaluate the patients' health conditions. Moreover, we have also considered the possibility of adding more advanced features such as integrating the body-attached sensor with portal service so that external user can monitor the patient wherever they are. The system will prompt the user to key in a username and password before the user can connect to the system. We believe that this research will result in better way to save someone's life during cardiac arrest and reduce the burden of lay rescuers to find an AED so that it can bring more practical and commercial value to our society.

\section{ACKNOWLEDGMENT}

The authors would like to thank faculty and student members of National Taipei University for providing equipment and material for this research.

\section{REFERENCES}

[1] The International Disaster Database, Centre for Research on the Epidemiology of Disasters - CRED: http://www.emdat.be/countryprofile.

[2] Eva Liu, Elyssa Wong. Emergency Ambulance Services. Research and Library Services Division Legislative Council Secretariat. June 1996.
[3] Kerber, Richard E; Becker, Lance B; Bourland, Joseph D; Cummins, Richard O; Hallstrom, Alfred P; Michos, Mary B; Nichol, Graham; Ornato, Joseph P; Thies, William H; White, Roger D; Zuckerman, Bram D (March 18, 1997). "Automatic External Defibrillators for Public Access Defibrillation". Circulation (American Heart Association) 95 (1677-1682):1677-82.

[4] Mohammad Arif, Hooman Samani, Chan-Yun Yang, and Yung-Yuan Chen, "Adaptation of mobile robot to intelligent vehicles," 2013 IEEE $4^{\text {th }}$ International Conference on Software Engineering and Service Science, Proceedings of, vol., no., pp.550-553, 23-25 May 2013.

[5] Casper, J.; Murphy, R.R., "Human-robot interactions during the robotassisted urban search and rescue response at the World Trade Center," Systems, Man, and Cybernetics, Part B: Cybernetics, IEEE Transactions on, vol.33, no.3, pp.367,385, June 2003.

[6] Matsuno, F.; Tadokoro, S., "Rescue Robots and Systems in Japan," Robotics and Biomimetics, 2004. ROBIO 2004. IEEE International Conference on, vol., no., pp.12,20, 22-26 Aug. 2004.

[7] Murphy, R.; Stover, S.; Pratt, K.; Griffin, C., "Cooperative Damage Inspection with Unmanned Surface Vehicle and Micro Unmanned Aerial Vehicle at Hurricane Wilma," Intelligent Robots and Systems, 2006 IEEE/RSJ International Conference on, vol., no., pp.9,9, Oct. 2006.

[8] Ho, Melissa, and Kevin Fall. "Poster: Delay tolerant networking for sensor networks." Proc. of IEEE Conference on Sensor and Ad Hoc Communications and Networks. 2004.

[9] Ohno, K.; Kawatsuma, S.; Okada, T.; Takeuchi, E.; Higashi, K.; Tadokoro, S., "Robotic control vehicle for measuring radiation in Fukushima Daiichi Nuclear Power Plant," Safety, Security, and Rescue Robotics (SSRR), 2011 IEEE International Symposium on, vol., no., pp.38,43, 1-5 Nov. 2011.

[10] Hayashi, T.; Kawamoto, H.; Sankai, Y., "Control method of robot suit HAL working as operator's muscle using biological and dynamical information," Intelligent Robots and Systems, 2005. (IROS 2005). 2005 IEEE/RSJ International Conference on, vol., no., pp.3063,3068, 2-6 Aug. 2005

[11] Jezernik, Sašo, et al. "Robotic orthosis lokomat: A rehabilitation and research tool." Neuromodulation: Technology at the Neural Interface 6.2 (2003): 108-115.

[12] Clement, R. G. E., K. E. Bugler, and C. W. Oliver. "Bionic prosthetic hands: a review of present technology and future aspirations." The surgeon 9.6 (2011): 336-340.

[13] H. A. Samani (2012) Book: Lovotics, Loving robots, ISBN: 3659155411 .

[14] Mukai, Toshiharu, et al. "Realization and Safety Measures of Patient Transfer by Nursing-Care Assistant Robot RIBA with Tactile Sensors." Journal of Robotics and Mechatronics 23.3 (2011): 360-369.

[15] Wada, Kazuyoshi, and Takanori Shibata. "Living with seal robots - its sociopsychological and physiological influences on the elderly at a care house." Robotics, IEEE Transactions on 23.5 (2007): 972-980.

[16] InTouch Health: RP-VITA ${ }^{\mathrm{TM}}$ robot. (2012) http://www.intouchhealth.com/products-and-services/products/rpvitarobot/

[17] Degen, Jeffrey W., et al. "CyberKnife stereotactic radiosurgical treatment of spinal tumors for pain control and quality of life." Journal of Neurosurgery: Spine2.5 (2005): 540-549.

[18] Bentas, Wassilios, et al. "Da Vinci robot assisted Anderson-Hynes dismembered pyeloplasty: technique and 1 year follow-up." World journal of urology 21.3 (2003): 133-138.

[19] Jaguar Robot. http://jaguar.drrobot.com/specification 4x4wArm.asp 


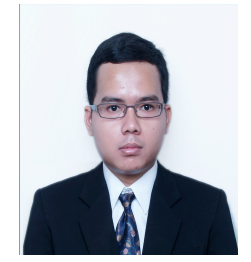

Mohammad Arif received the B.IT. degree in Industrial Computing from Universiti Kebangsaan Malaysia, Malaysia, in 2012. Since 2012, he is currently pursuing the M.Sc. degree in Electrical Engineering from National Taipei University. His current research interest is in mobile robot applications.

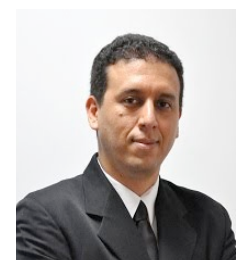

Hooman Samani received the $\mathrm{PhD}$ degree from the Graduate School for Integrative Science and Engineering, Electrical \& Computer Engineering Department, National University of Singapore. He is currently an Asst. Professor at the Department of Electrical Engineering, College of Electrical Engineering and Computer Science, National Taipei University, Taiwan. 